\title{
Gold Nanoparticles: Synthesis, Stability Test, and Application for the Rice Growth
}

\author{
Aiwu Wang, ${ }^{1,2,3}$ Hoi Pong $\mathrm{Ng}^{2}{ }^{2}$ Yi Xu, ${ }^{2}$ Yuyu Li, ${ }^{2}$ Yuhong Zheng, ${ }^{1}$ Jingping Yu, \\ Fugui Han, ${ }^{1}$ Feng Peng, ${ }^{1}$ and $\mathrm{Li} \mathrm{Fu}^{4}$ \\ ${ }^{1}$ Institute of Botany, Jiangsu Province and Chinese Academy of Sciences, \\ Nanjing Botanical Garden, Memorial Sun Yat-Sen, Nanjing 210014, China \\ ${ }^{2}$ Department of Physics and Materials Science and Centre for Functional Photonics (CFP), City University of Hong Kong, \\ 88 Tat Chee Avenue, Kowloon, Hong Kong \\ ${ }^{3}$ Faculty of Science and Technology, University of Macau, Macau \\ ${ }^{4}$ Department of Chemistry and Biotechnology, Faculty of Science, Engineering and Technology, Swinburne University of Technology, \\ Hawthorn, VIC 3122, Australia
}

Correspondence should be addressed to Yuhong Zheng; friend266@163.com

Received 8 July 2014; Accepted 10 August 2014; Published 2 September 2014

Academic Editor: Tifeng Jiao

Copyright (C) 2014 Aiwu Wang et al. This is an open access article distributed under the Creative Commons Attribution License, which permits unrestricted use, distribution, and reproduction in any medium, provided the original work is properly cited.

In today's science, with the use of nanotechnology, nanomaterials, which behave very differently from the bulk solid, can be made. One of the capable uses of nanomaterials is bioapplications which make good use of the specific properties of nanoparticles. However, since the nanoparticles will be used both in vivo and in vitro, their stability is an important issue to the scientists, concern. In this dissertation, we are going to test the stability of gold nanoparticles in a number of media including the biocompatible medium and their behaviors will be illustrated in terms of optical properties change and aggregation degree. Herein, we report the synthesis of gold nanoparticles of different shapes and applications for the rice growth with significant difference. The gold nanoparticles can inhibit the elongation of rice root without inhibiting the germination of rice seeds.

\section{Introduction}

Gold nanoparticles (AuNPs) are also called colloidal gold or gold colloids. Similar to semiconductors, when metals decrease their size from bulk to the nanoscale, they also experience quantum confinement effect [1]. The conduction band electrons of the metal nanoparticles will resonate with the electromagnetic field, that is, light, and hence cause light absorption; this phenomenon is known as surface plasmon resonance [2-5]. Surface plasmon resonance was explained by Mie in 1908 [6]. However, at that time, Mie's theory could only be applied on metal nanoparticles which are much smaller than wavelength of light (about $25 \mathrm{~nm}$ ) due to the assumption of the theory. The theory shows that plasmon absorption is size independent [7-9]. By the full expression of Mie's theory, it can be applied to large metal nanoparticles $(>25 \mathrm{~nm})$ and can show size dependence of plasmon absorption [1013].

Since the plasmon absorption maximum of AuNPs is size dependent [14-19], by tuning their size, their absorption maximum and color can be tuned.

Although gold is one of the most stable elements in the world, when AuNPs with diameter smaller than $5 \mathrm{~nm}$ are deposited on select metal oxides, they exhibit extraordinary selectivities and/or activities in some reactions such as combustion of carbon monoxide and saturated hydrocarbons [20] which show catalytic property.

In the presence of light, the conduction band electrons of AuNPs oscillate due to surface plasmon resonance. The oscillating electrons interact with the crystal lattice of AuNPs and transfer thermal energy to the lattice. Thus, AuNPs are heated up and can further dissipate their thermal energy to the surrounding medium to achieve the heating effect [21]. 


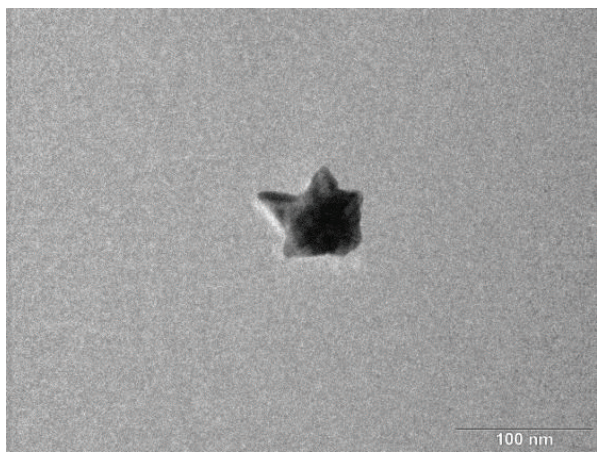

(a)

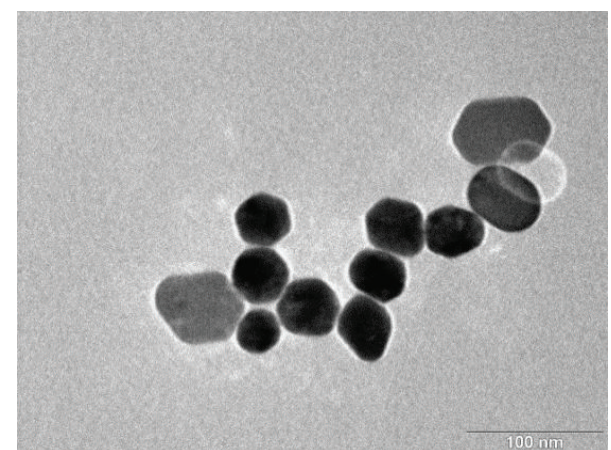

(b)

FIgure 1: (a) TEM image of CTAB AuNPs. (b) TEM image of citrate AuNPs.

\section{Experimental Parts}

2.1. Synthesis of Citrate Gold Nanoparticles. First, $25 \mathrm{~mL}$ of water and a magnetic stir bar were added into a flask and the flask was placed on stirrer and heated to $100^{\circ} \mathrm{C}$. Second, $52.4 \mathrm{mg}$ of $\mathrm{HAuCl}_{4}$ was weighed in a dry $100 \mathrm{~mL}$ flask and was placed on a stirrer. Then, $75 \mathrm{~mL}$ of water was added and the gold salt was dissolved under stirring. After all of the gold salt was dissolved, $10 \mathrm{~mL}$ of the solution was poured into the hot water flask. Next, citrate stock solution was prepared by dissolving $506.3 \mathrm{mg} \mathrm{Na} \mathrm{C}_{6} \mathrm{H}_{5} \mathrm{O}_{7} \cdot 2 \mathrm{H}_{2} \mathrm{O}$ in $75 \mathrm{~mL}$ of Milli-Q water. After the gold solution was boiled, $4 \mathrm{~mL}$ of citrate stock solution was added and the solution was boiled for 1.5 hours. After 1.5 hours, the flask was put onto a table and we waited till it cools down. Finally, the solution was stored in a $50 \mathrm{~mL}$ test tube at room temperature without any special treatment.

2.2. Synthesis of CTAB Gold Nanoparticles. First, $100 \mathrm{~mL}$ of $0.1 \mathrm{M} \mathrm{CTAB}, 10 \mathrm{~mL}$ of $0.01 \mathrm{M} \mathrm{HAuCl}_{4}, 5 \mathrm{~mL}$ of $0.1 \mathrm{M}$ of ascorbic acid, and $10 \mathrm{~mL}$ of $0.01 \mathrm{M}$ of $\mathrm{AgNO}_{3}$ solution were prepared. The CTAB solution was heated to $30^{\circ} \mathrm{C}$ in a water bath. Then, $3 \mathrm{~mL}$ of $0.1 \mathrm{M} \mathrm{CTAB}$ was added into a test tube. $1.748 \mathrm{~mL}$ of Milli-Q water, $200 \mu \mathrm{L} \mathrm{HAuCl}_{4}$, and $32 \mu \mathrm{L}$ ascorbic acid were added one by one. The solution was mixed gently after each addition. Next, $20 \mu \mathrm{L}$ of $0.01 \mathrm{M}$ silver nitrate was added into the test tube and we waited for 4 seconds before gently mixing it. After the solution was prepared, it was placed inside a $30^{\circ} \mathrm{C}$ water bath and we waited for 3 hours for complete reaction. Finally, the solution was washed twice by centrifuging at $4500 \mathrm{rmp}$ for $5 \mathrm{~min}$ each time and then it was stored at room temperature.

\section{Results and Discussion}

From Figure 1(a), we can observe that the CTAB AuNPs are star shaped with the longest dimension in between 80 and $90 \mathrm{~nm}$. So it is expected that we can observe two peaks or one peak and one shoulder from the absorption spectra.

Figure 1(b) shows that the citrate AuNPs are almost spherical in shape with the diameter from about 30 to $50 \mathrm{~nm}$. The larger, brighter, and irregular shaped particles on the left hand side should be the precipitated citrate.

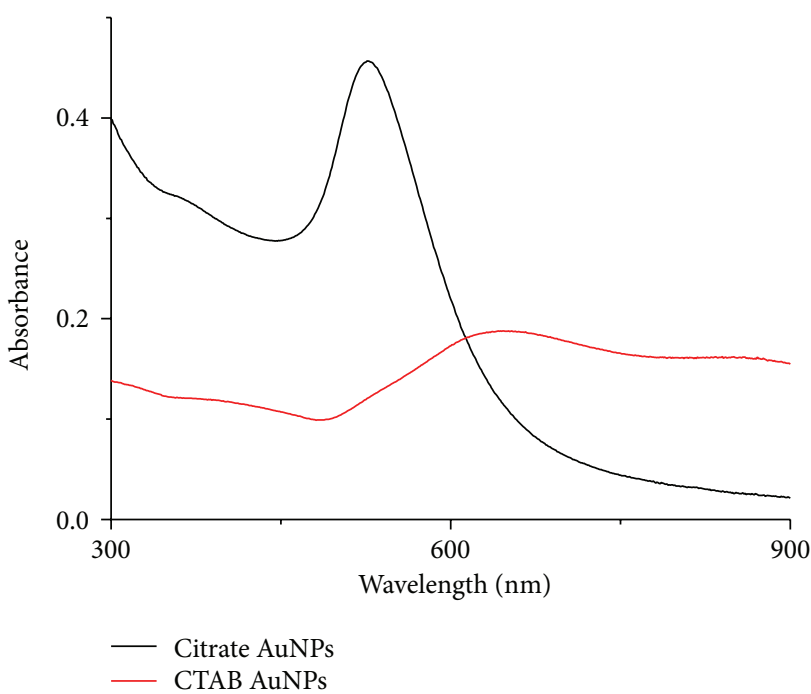

Figure 2: Absorption spectra of citrate and CTAB AuNPs.

From Figure 2, we can observe that citrate AuNPs have a peak and a shoulder while citrate AuNPs only have one peak.

3.1. Stability of CTAB AuNPs in Different Media. Table 1 showed the absorption peak position and intensity of CTAB AuNPs.

3.1.1. CTAB AuNPs in Milli-Q Water and Biobuffer. The absorption intensities of CTAB AuNPs in both Milli-Q water and biobuffer are very stable within the time of inspection. And their absorption peak positions were very stable too. These mean that CTAB AuNPs were very stable in both Milli$\mathrm{Q}$ water and biobuffer.

3.1.2. CTAB AuNPs in pH 4, pH 7, pH 8, and pH 10 Buffers. CTAB AuNPs behave similarly in $\mathrm{pH} 4, \mathrm{pH} 7, \mathrm{pH} 8$, and $\mathrm{pH} 10$ buffers. They showed a rapid drop in absorption intensities in the first 15 minutes and decreased slowly afterwards. Meanwhile, their absorption peak positions also demonstrated a blue shift in the first 15 minutes and remained unchanged up 
TABLE 1: Absorption peak position and intensity variation of CTAB AuNPs with time in different media.

\begin{tabular}{|c|c|c|c|c|c|}
\hline & Hour & Position 1 & Position 2 & Intensity $1 \%$ & Intensity $2 \%$ \\
\hline \multirow{7}{*}{ Water } & 0 & 639 & $840-$ & 100 & 100 \\
\hline & 0.25 & 639 & $840-$ & 100 & 100 \\
\hline & 0.5 & 639 & $840-$ & 100 & 100 \\
\hline & 1 & 639 & $840-$ & 100 & 100 \\
\hline & 2 & 639 & $840-$ & 100 & 100 \\
\hline & 72 & 639 & $840-$ & 94 & 93 \\
\hline & 168 & 639 & $840-$ & 102 & 102 \\
\hline \multirow{7}{*}{ pH 4 buffer } & $\mathbf{0}$ & 640 & $852.2-$ & 90 & 104 \\
\hline & 0.25 & 617 & $852.2-$ & 63 & 87 \\
\hline & 0.5 & 617 & $852.2-$ & 57 & 79 \\
\hline & 1 & 617 & $852.2-$ & 53 & 73 \\
\hline & 2 & 617 & $852.2-$ & 48 & 66 \\
\hline & 72 & $640-$ & $852.2-$ & 7 & 9 \\
\hline & 168 & $640-$ & $852.2-$ & 25 & 29 \\
\hline \multirow{7}{*}{ pH 7 buffer } & $\mathbf{0}$ & 623 & 851 & 80 & 98 \\
\hline & 0.25 & 620 & $851-$ & 57 & 74 \\
\hline & 0.5 & 620 & $851-$ & 53 & 67 \\
\hline & 1 & 620 & $851-$ & 50 & 63 \\
\hline & 2 & 620 & $851-$ & 44 & 57 \\
\hline & 72 & $623-$ & $851-$ & 1 & 2 \\
\hline & 168 & $623-$ & $851-$ & 14 & 16 \\
\hline \multirow{7}{*}{ pH 8 buffer } & 0 & 629 & 850 & 84 & 105 \\
\hline & 0.25 & 607 & $850-$ & 56 & 78 \\
\hline & 0.5 & 607 & $850-$ & 52 & 70 \\
\hline & 1 & 607 & $850-$ & 48 & 65 \\
\hline & 2 & 607 & $850-$ & 43 & 60 \\
\hline & 72 & $629-$ & $850-$ & 1 & 1 \\
\hline & 168 & $629-$ & $850-$ & 18 & 21 \\
\hline \multirow{7}{*}{ pH 10 buffer } & 0 & 631 & 836 & 90 & 97 \\
\hline & 0.25 & 611 & $836-$ & 53 & 71 \\
\hline & 0.5 & 611 & $836-$ & 47 & 64 \\
\hline & 1 & 611 & $836-$ & 42 & 53 \\
\hline & 2 & 611 & $836-$ & 36 & 48 \\
\hline & 72 & $631-$ & $836-$ & 2 & 2 \\
\hline & 168 & $631-$ & $836-$ & 17 & 21 \\
\hline \multirow{8}{*}{ Biobuffer } & 0 & 560 & 584 & 61 & 68 \\
\hline & 0.25 & 560 & 585 & 60 & 67 \\
\hline & 0.5 & 560 & 586 & 60 & 66 \\
\hline & 1 & 560 & 587 & 59 & 67 \\
\hline & 2 & 560 & 588 & 59 & 67 \\
\hline & 3 & 560 & 588 & 59 & 67 \\
\hline & 72 & 560 & $584-$ & 59 & 65 \\
\hline & 168 & 560 & $584-$ & 57 & 64 \\
\hline
\end{tabular}

${ }^{* *}$ For the number with "-_" behind means no clear peak can be observed and we measure the intensity at that wavelength.

to 2 hours. Subsequently, their absorption intensities dropped to a few intensity percentages and no peaks can be observed at the third day. However, their absorption intensities increased again at the seventh day; this may be because the CTAB
AuNPs form larger aggregates than those at the third day; therefore, scattering of the CTAB AuNPs increased. These represented that CTAB AuNPs are not stable in $\mathrm{pH} 4, \mathrm{pH} 7$, $\mathrm{pH}$ 8, and $\mathrm{pH} 10$ buffers. 
TABLE 2: Zeta potential and size of citrate AuNPs in Milli-Q water.

\begin{tabular}{|c|c|c|c|c|}
\hline Time (hour) & Zeta potential $(\mathrm{mV})$ & Zeta potential (mV) (second measure) & Size $1(\mathrm{~nm})$ & Size $2(\mathrm{~nm})$ \\
\hline 0 & -27.5 & - & 5.6 & 50.8 \\
\hline 1 & -16.1 & - & 5.6 & 50.8 \\
\hline 2 & -19.0 & - & 5.6 & 50.8 \\
\hline 3 & -17.1 & -32.2 & 5.6 & 50.8 \\
\hline 24 & -37.5 & - & 5.6 & 50.8 \\
\hline 72 & -33.4 & - & 5.6 & 50.8 \\
\hline 168 & -39.9 & - & 5.6 & 50.8 \\
\hline
\end{tabular}

"_" means no measurement has been done.

To sum up, CTAB AuNPs were very stable in Milli-Q water and biobuffer; this indicated that they can be used for bioapplications. But they were unstable in $\mathrm{pH} 4, \mathrm{pH} 7, \mathrm{pH} 8$, and $\mathrm{pH} 10$ buffers.

3.2. Stability of Citrate AuNPs in Different Media. Citrate has a shorter carbon length than CTAB. As there is no big difference in peak position, only the absorption peak intensity variation was shown in Figure 3.

3.2.1. Citrate AuNPs in Milli-Q Water and Biobuffer. We can observe that the absorption peak intensities of citrate AuNPs in Milli-Q water and biobuffer were very stable within the period of inspection. And their absorption peak position in Milli-Q water and biobuffer was always the same in the experimental time. These represented that citrate AuNPs were very stable in Milli-Q water and biobuffer.

3.2.2. Citrate AuNPs in $p H 4, p H 7$, and $p H 10$ Buffers. Citrate AuNPs perform similarly in $\mathrm{pH} 4, \mathrm{pH}$ 7, and $\mathrm{pH} 10$ buffers. All of them showed fast drop in absorption peak intensity in the first 15 minutes and then dropped slowly thereafter. At the fourth day, they only have about $5 \%$ absorption peak intensity. Moreover, the absorption peak position of $\mathrm{pH} 4$ buffer showed small red shift from $524 \mathrm{~nm}$ at 0 minutes to $533 \mathrm{~nm}$ at 2 hours which may be because of the increase in aggregate size and showed a large blue shift to $507 \mathrm{~nm}$ at the fourth day; this may be due to the degradation of citrate AuNPs which have a smaller size than their size at 0 minutes. But the absorption peak positions of citrate AuNPs in $\mathrm{pH}$ 7 and pH 10 buffers were almost the same within the first 2 hours and then no peaks can be found afterwards. These phenomena mean that citrate AuNPs are unstable in $\mathrm{pH} 4$, $\mathrm{pH} 7$, and $\mathrm{pH} 10$ buffers.

3.2.3. Citrate AuNPs in $p H 8$ Buffer. The absorption peak intensity of citrate AuNPs in $\mathrm{pH} 8$ buffer decreases slowly within the first 2 hours and then drops to about $40 \%$ intensity thereafter. And their absorption peak position was almost the same with the experimental time. Thus, they are unstable in pH 8 buffer in terms of absorption peak intensity.

To sum up, citrate AuNPs were very stable in Milli-Q water and biobuffer which made them able to be used for bioapplications. But they were unstable in $\mathrm{pH} 4, \mathrm{pH} 7, \mathrm{pH} 8$,

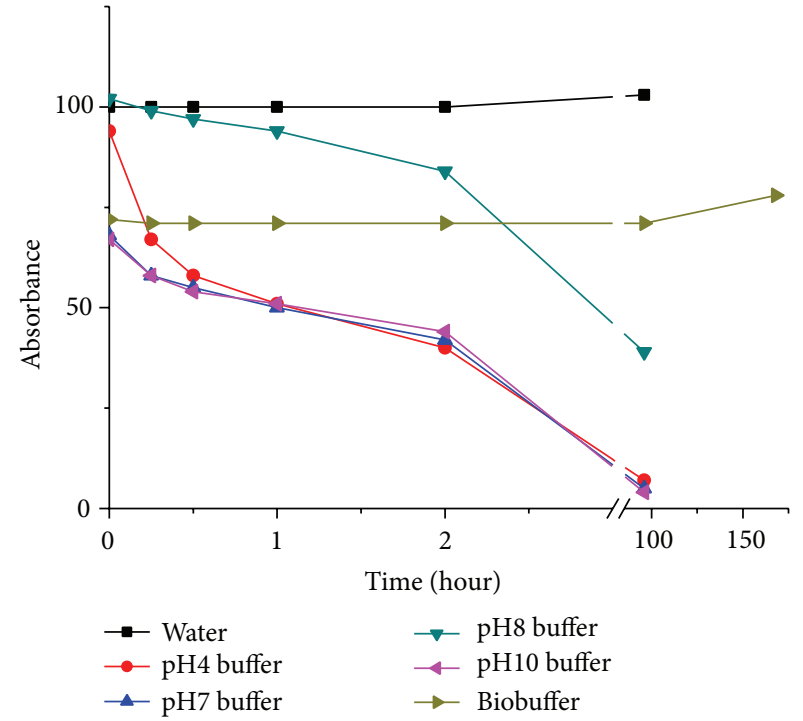

FIGURE 3: Absorption peak intensity of citrate AuNPs in different media varies with time.

and $\mathrm{pH} 10$ buffers. Compared with the first absorption peak of CTAB AuNPs, both of them showed similar behavior for all buffers, that is, being unstable in $\mathrm{pH} \mathrm{4,} \mathrm{pH} 7, \mathrm{pH} 8$, and $\mathrm{pH} 10$ buffers but very stable in Milli-Q water and biobuffer, which allows them to be used for bioapplications.

3.3. Zeta Potential and Size of Citrate AuNPs. For the citrate AuNPs in Milli-Q water, referring to Table 2, it is noted that the zeta potential is very good at 0 hours but decreased with time in the first 3 hours. At the same time, the color of the citrate AuNPs in the zeta cell for zeta potential measurement fades out after each measurement. This may be because in the measurement of zeta potential the citrate AuNPs adhere to the surface of the gold-plated electrodes.

But if the citrate AuNPs in the zeta cell were replaced by the spare solution that has not been used for measurement before, a good zeta potential can be observed again, that is, zeta potential of second measurement of the 3 hours and measurement after 3 hours.

And the size of the citrate AuNPs was the same for all time ranges. These were in good agreement with the absorption spectrum. 

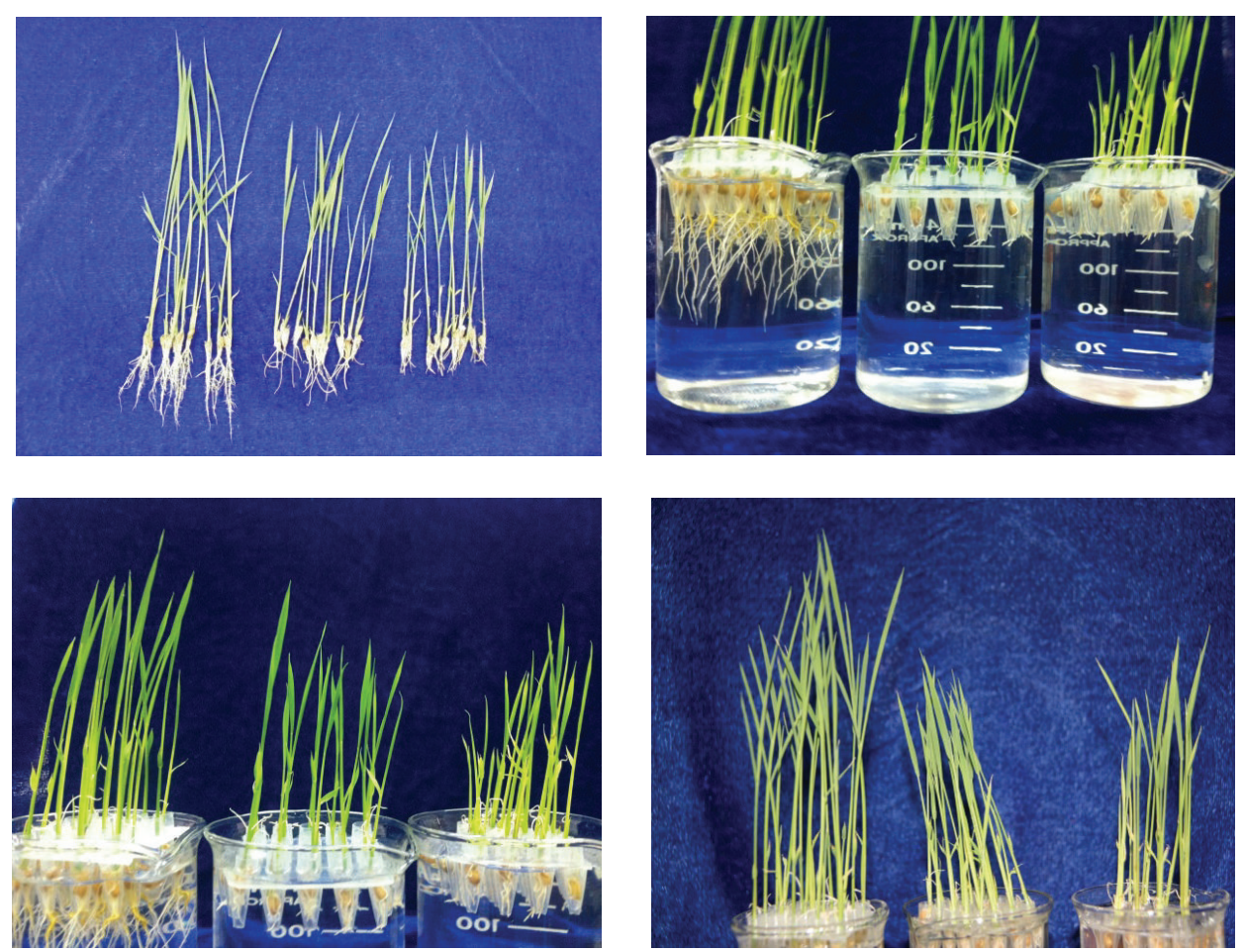

FIGURE 4: Images of rice: no gold nanoparticles, $5 * 10^{-6} \mathrm{M}$ gold nanoparticles, and $5 * 10^{-8} \mathrm{M}$ gold nanoparticles.

TABLE 3: Zeta potential and size of citrate AuNPs in pH 4 buffer.

\begin{tabular}{lcc}
\hline Time (hour) & Zeta potential $(\mathrm{mV})$ & Size $(\mathrm{nm})$ \\
\hline 0 & -46.1 & 342.0 \\
1 & -39.4 & 825.0 \\
2 & -38.8 & 531.2 \\
3 & -36.0 & 255.0 \\
24 & -33.0 & 295.3 \\
72 & -29.9 & 712.4 \\
168 & -13.9 & 141.8 \\
\hline
\end{tabular}

From Table 3, the zeta potential of the citrate AuNPs in $\mathrm{pH} 4$ buffer was evenly more negative than that in Milli-Q water. But aggregation happened because some components in the buffer adsorb on the surface of the citrate AuNPs and crosslinks form in between AuNPs. These results agreed with the absorption spectrum.

According to Table 4, the zeta potential of citrate AuNPs in biobuffer was about 5 times lower than that in Milli-Q water at 0 hours. And size 1 was due to the biobuffer and size 2 was the size of the citrate AuNPs which was almost the same within the measurement period. As the zeta potential and size were stable with time, so it was in a good agreement with the absorption spectrum.

To sum up, the zeta potentials of citrate AuNPs in Milli$\mathrm{Q}$ water and biobuffer were very stable while those in $\mathrm{pH}$ 4 buffer were decreasing during the experiment. Moreover, their size in Milli-Q water and biobuffer was also stable for
TABLE 4: Zeta potential and size of citrate AuNPs in biobuffer.

\begin{tabular}{lccc}
\hline Time (hour) & Zeta potential $(\mathrm{mV})$ & Size 1 $(\mathrm{nm})$ & Size 2 $(\mathrm{nm})$ \\
\hline 0 & -9.54 & 10.1 & 78.8 \\
1 & -10.6 & 5.6 & 122.4 \\
2 & -10.2 & 10.1 & 91.3 \\
3 & -10.5 & 10.1 & 78.8 \\
24 & -11.4 & 13.5 & 78.8 \\
72 & -11 & 11.7 & 78.8 \\
168 & -10.6 & 10.1 & 78.8 \\
\hline
\end{tabular}

all times within the experimental period while they showed aggregation in $\mathrm{pH} 4$ buffer.

3.4. Application for the Rice Growth. As citrate AuNPs were rather stable in water and biobuffer, they were used in the application for the rice growth.

From Figure 4 and Table 5 we can observe that with the AuNPs in a rather low concentration the rice roots stopped growing and the height of seedlings got influenced too; however the presence of AuNPs did not inhibit the germination of rice seed.

\section{Conclusions}

In Milli-Q water, both AuNPs are very stable. In $\mathrm{pH} 4$, $\mathrm{pH} 7, \mathrm{pH} 8$, and $\mathrm{pH} 10$ buffers, both of them are unstable. 
TABLE 5: The height, number, and length for rice.

\begin{tabular}{lcccc}
\hline & The height of seedlings & The number of roots & The length of roots & $\begin{array}{c}\text { The ratio of root length and } \\
\text { seedling height }\end{array}$ \\
\hline CK & $17.78 \pm 1.82 \mathrm{Aa}$ & $7.50 \pm 1.80 \mathrm{Aa}$ & $3.39 \pm 1.35 \mathrm{Aa}$ & $0.191 \mathrm{Aa}$ \\
$\mathrm{T} 1$ & $10.79 \pm 3.03 \mathrm{Bb}$ & $6.90 \pm 1.29 \mathrm{Aa}$ & $1.90 \pm 0.74 \mathrm{Bb}$ & $0.176 \mathrm{Bb}$ \\
T2 & $11.60 \pm 0.15 \mathrm{Bb}$ & $7.30 \pm 1.42 \mathrm{Aa}$ & $1.67 \pm 0.50 \mathrm{Bb}$ & $0.144 \mathrm{Bb}$ \\
\hline
\end{tabular}

$\mathrm{Aa} \mathrm{Bb}$ are Significant symbols which commonly used in the Data Analysis. Use the uppercase and lowercase letters (A a) due to the significant results.

In biobuffer, they were very stable which indicated that both CTAB and citrate AuNPs can be used for bioapplications.

To conclude, we found important information for the practical use of nanoparticles in bioapplications. Both CTAB and citrate AuNPs are also very good for bioapplications. And citrate AuNPs can inhibit the elongation of rice root to a large degree without inhibiting the germination of rice seed.

\section{Conflict of Interests}

The authors declare that there is no conflict of interests regarding the publication of this paper.

\section{Acknowledgments}

The authors thank the support from Institute of Botany, Jiangsu Province, Chinese Academy of Sciences, Nanjing Botanical Garden, Department of Physics and Materials Science and Centre for Functional Photonics (CFP), City University of Hong Kong, Department of Chemistry and Biotechnology, Faculty of Science and Technology, University of Macau, Macau Faculty of Science, Engineering and Technology, and Swinburne University of Technology.

\section{References}

[1] T. Vossmeyer, L. Katsikas, M. Giersig, I. G. Popovic, K. D. Chemseddine, and A. Eychmuller, "Simultaneous control of nanocrystal size and nanocrystal-nanocrystal separation in CdS nanocrystal assembly," The Journal of Physical Chemistry, vol. 65, no. 4, pp. 565-570, 1994.

[2] H. S. Choi, W. Liu, P. Misra et al., "Renal clearance of quantum dots," Nature Biotechnology, vol. 25, no. 2, pp. 1165-1170, 2007.

[3] A. L. Rogach, A. Kornowski, M. Gao, A. Eychmüller, and H. Weller, "Synthesis and characterization of a size series of extremely small thiol-stabilized CdSe nanocrystals," The Journal of Physical Chemistry B, vol. 103, no. 16, pp. 3065-3069, 1999.

[4] A. L. Rogach, L. Katsikas, A. Kornowski, S. Dangsheng, A. Eychmüller, and H. Weller, "Synthesis and characterization of thiol-stabilized CdTe nenocrystals," Berichte der Bunsengesellschaft fur Physikalische Chemie, vol. 100, no. 11, pp. 1772-1778, 1996.

[5] A. L. Rogach, "Fluorescence energy transfer in hybrid structures of semiconductor nanocrystals," Nano Today, vol. 6, no. 4, pp. 355-365, 2011.

[6] A. Rogach, S. Kershaw, M. Burt et al., "Optical properties of semiconductor nanostructures," Advanced Materials, vol. 11, pp. $552-555,1999$.
[7] U. Resch-Genger, M. Grabolle, S. Cavaliere-Jaricot, R. Nitschke, and T. Nann, "Quantum dots versus organic dyes as fluorescent labels," Nature Methods, vol. 5, no. 9, pp. 763-775, 2008.

[8] M. Green, "Semiconductor quantum dots as biological imaging agents," Angewandte Chemie, vol. 43, no. 32, pp. 4129-4131, 2004.

[9] J. K. Jaiswal and S. M. Simon, "Potentials and pitfalls of fluorescent quantum dots for biological imaging," Trends in Cell Biology, vol. 14, no. 9, pp. 497-504, 2004.

[10] M. Bruchez Jr., M. Moronne, P. Gin, S. Weiss, and A. P. Alivisatos, "Semiconductor nanocrystals as fluorescent biological labels," Science, vol. 281, no. 5385, pp. 2013-2016, 1998.

[11] A. M. Derfus, W. C. W. Chan, and S. N. Bhatia, "Probing the cytotoxicity of semiconductor quantum dots," Nano Letters, vol. 4, no. 1, pp. 11-18, 2004.

[12] S. T. Selvan, T. T. Tan, and J. Y. Ying, "Robust, non-cytotoxic, silica-coated CdSe quantum dots with efficient photoluminescence," Advanced Materials, vol. 17, no. 13, pp. 1620-1625, 2005.

[13] T. Vossmeyer, L. Katsikas, M. Giersig et al., "CdS nanoclusters: synthesis, characterization, size dependent oscillator strength, temperature shift of the excitonic transition energy, and reversible absorbance shift," Journal of Physical Chemistry, vol. 98, no. 31, pp. 7665-7673, 1994.

[14] M. V. Khodakovskaya, B.-S. Kim, J. N. Kim et al., "Carbon nanotubes as plant growth regulators: effects on tomato growth, reproductive system, and soil microbial community," Small, vol. 9, no. 1, pp. 115-123, 2013.

[15] S. Wu, J. Dou, J. Zhang, and S. Zhang, "A simple and economical one-pot method to synthesize high-quality water soluble CdTe QDs," Journal of Materials Chemistry, vol. 22, no. 29, pp. 1457314578, 2012.

[16] A. L. Rogach, T. Franzl, T. A. Klar et al., "Aqueous synthesis of thiol-capped CdTe nanocrystals: state-of-the-art," The Journal of Physical Chemistry C, vol. 111, no. 40, pp. 14628-14637, 2007.

[17] P. Alivisatos, "The use of nanocrystals in biological detection," Nature Biotechnology, vol. 22, no. 1, pp. 47-52, 2004.

[18] C. A. Mirkin, R. L. Letsinger, R. C. Mucic, and J. J. Storhoff, "A DNA-based method for rationally assembling nanoparticles into macroscopic materials," Nature, vol. 382, no. 6592, pp. 607609, 1996.

[19] J. D. Gosnell, M. A. S. Schreuderb, M. J. Bowers II, S. J. Rosenthal, and S. M. Weiss, "Cadmium selenide nanocrystals as white-light phosphors," in 6th International Conference on Solid State Lighting, vol. 6337 of Proceedings of the SPIE, p. 63370A, 2006.

[20] J.-H. Kim, Y. Lee, E.-J. Kim et al., "Exposure of iron nanoparticles to arabidopsis thaliana enhances root elongation by triggering cell wall loosening," Environmental Science \& Technology, vol. 48, no. 6, pp. 3477-3485, 2014.

[21] M. Haruta, "Size- and support-dependency in the catalysis of gold," Catalysis Today, vol. 36, no. 2, pp. 153-166, 1997. 

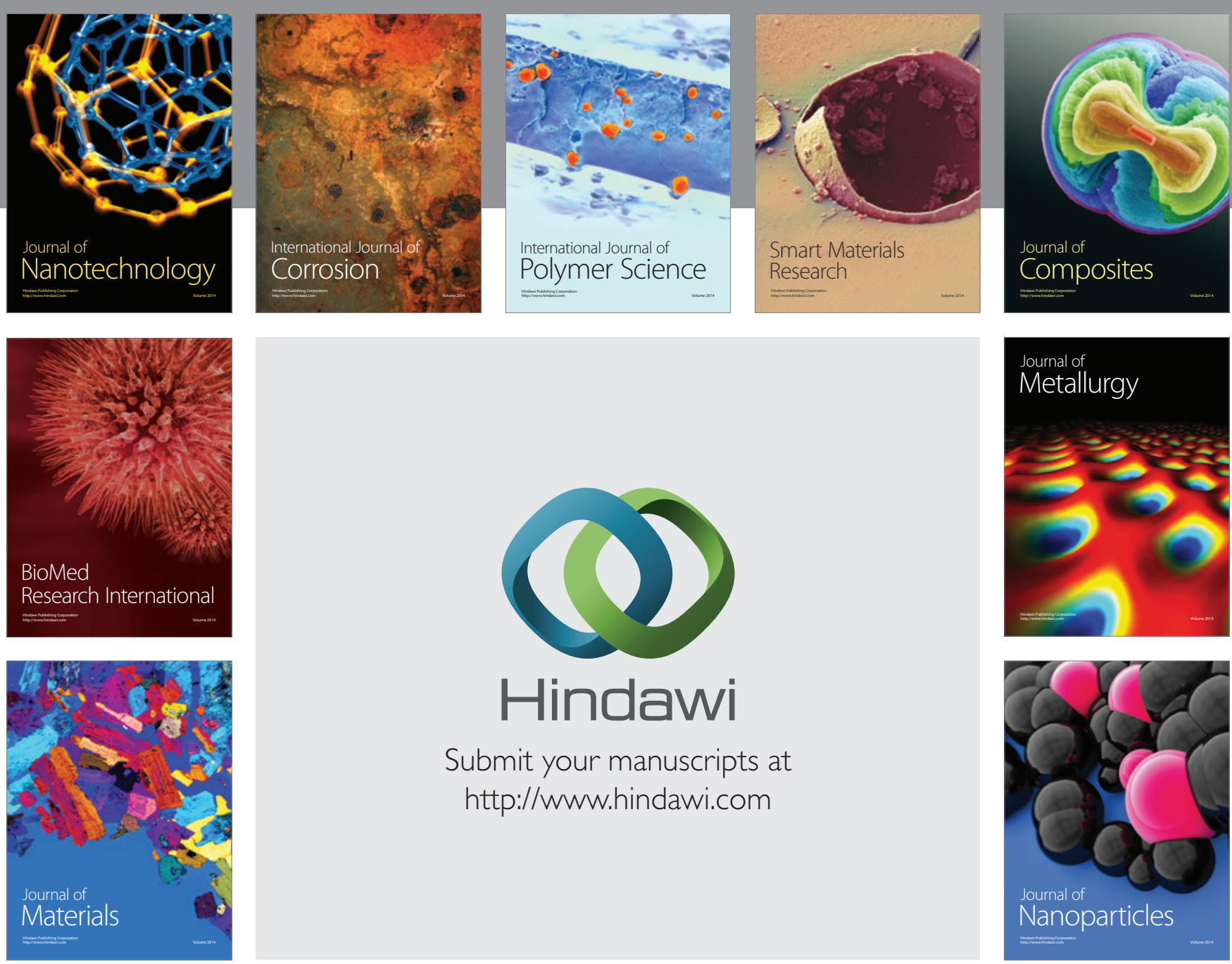

Submit your manuscripts at http://www.hindawi.com
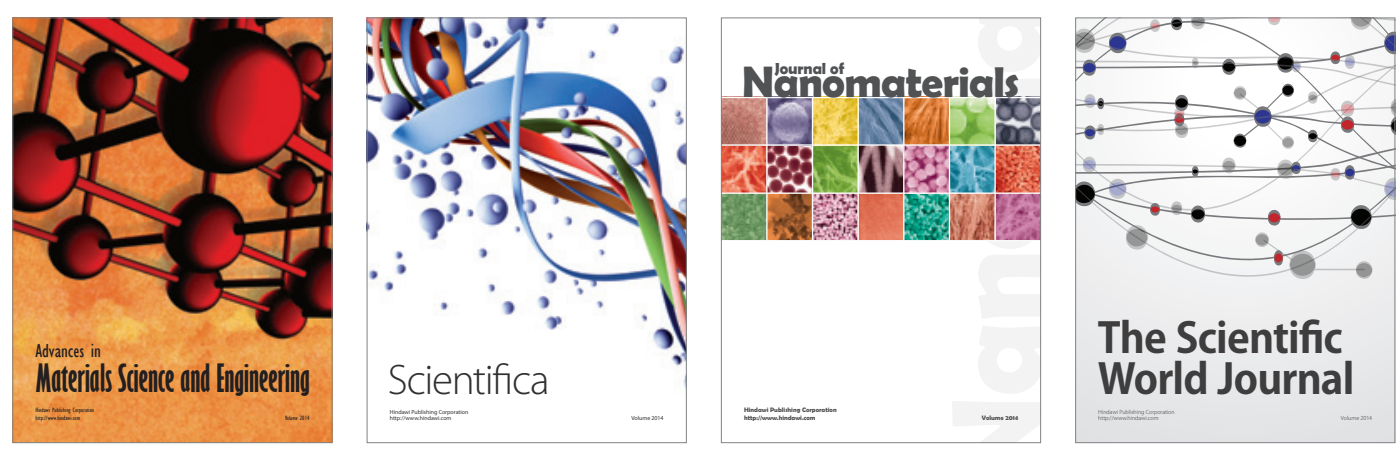

\section{The Scientific World Journal}
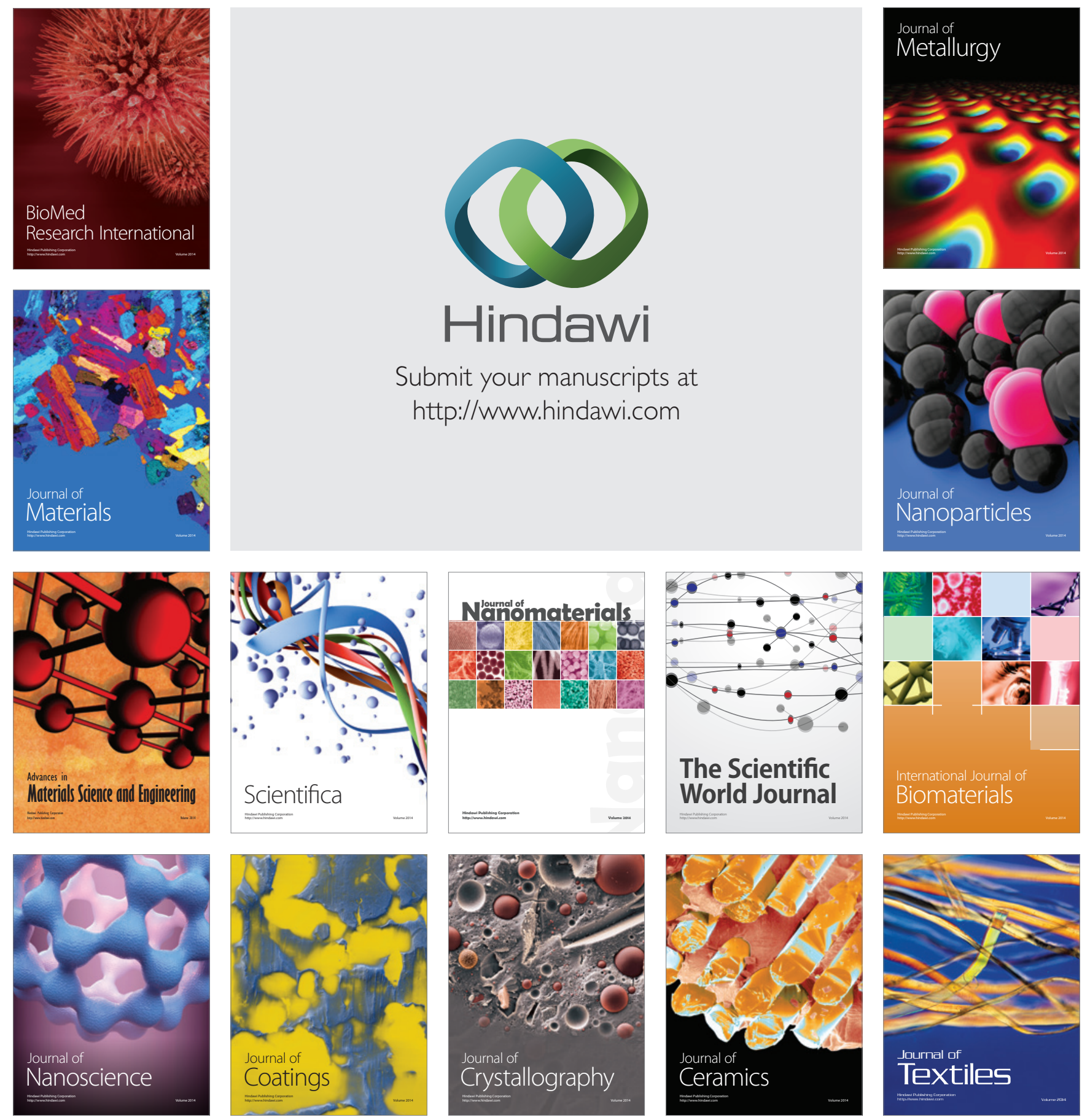